\title{
EL FUTURO DE LAS PYMEs EN LA GLOBALIZACIÓN
}

Fidel Antonio Vásquez Ramoz'

\section{RESUMEN}

En la actualidad, la empresa que no se integra a la globalización está destinada a desaparecer, asimismo esta integración exige que las empresas estén en constante movimiento tecnológico e innovando métodos y sistemas de producción y organización, dentro de estos sistemas están LA PROSPECTIVA TECNOLOGICA Y EL CUADRO DE MANDO ESTRATÉGICO (BALANCED SCORECARD), estas son nuevas HERRAMIENTAS PARA EL CRECIMIENTO Y DESARROLLO ESTRATÉGICO de las PYMEs.

LA PROSPECTIVA TECNOLÓGICA, son mediciones que se realizan prospectivamente, para analizar los comportamientos futuros de los consumidores finales y coadyuvan al desarrollo de las políticas gubernamentales y empresariales. Las PYMEs, orientando la inversión y la producción, El empleo de la Prospectiva Tecnológica ha permitido a medianas economías tener altas tasas de crecimiento económico, luego de la ejecución del Ejercicio Nacional de Prospectiva Tecnológica.

Estas mediciones se realizan siguiendo diversas metodologías que se pueden emplear en un Ejercicio de Prospectiva Tecnológica. Las más empleadas son:

- Exploración del Entorno

- Análisis de Tendencias

- Análisis Morfológico

- Árboles de Pertinencia

- Encuesta Delphi

- Análisis de Jerarquía de Procesos

- Matriz de Impacto Cruzado

- Planeamiento de Escenarios

El Cuadro de Mando Estratégico es un enfoque que plantea ver a la organización desde 4 perspectivas distintas e interrelacionadas.

La Perspectiva desde el Punto de Vista Financiero

La Perspectiva desde el Punto de Vista dei Cliente

La Perspectiva de los Procesos Internos de la Organización

La Perspectiva de la Innovación, Desarrollo y el Crecimiento

La idea es que dentro de estas visiones se establezcan diversos indicadores de gestión, que permitan hacer un seguimiento adecuado dei desempeño en cada una de estas perspectivas y sus relaciones con las demás y en consecuencia, lograr un sistema de medición integral y permanente dei desempeño de la organización como un todo; integrando a la vez, la misión, visión, objetivos, iniciativas estratégicas. sus indicadores de gestión y las metas a cumplir. 


\section{INTRODUCCIÓN}

¿Qué pasará con las PYMEs peruanas al final de esta década? Esta es una pregunta que nos hacemos todos los que estamos vinculados con el desarrollo de este estratégico sector de la economía de nuestro país.

Como no podemos acudir a una bola de cristal para conocer el futuro que les espera a las PYMEs, debemos encontrar un medio racional y científico que nos permita vislumbrar los escenarios futuros que enfrentarán las PYMEs al final de esta década. Ese método es la Prospectiva Tecnológica.

Durante los últimos años se han venido desarrollando un conjunto de herramientas para el mejoramiento del desempeño estratégico organizacional. Un Ejercicio de Prospectiva Tecnológica cuyo foco de atención sean las PYMEs se convierte en una necesidad impostergable.

Diversos enfoques han sido empleados para ello. En el ámbito sistémico, en el cual nos desenvolvemos, son conocidos los aportes del Modelo de Sistema Viable del Prof. $\mathrm{S}$. Beer, la Metodología de Sistemas Blandos (Soft Systems) del Prof. PB. Checkland, los aportes en Interactive Management del Prof. J. H. Warfield, la Dinámica de Sistemas y las 5 Disciplinas de J. Forrester y P Senge, Strategic Interactive Management de R. Ackoff, entre otros.

En el campo del pensamiento estratégico, es importante mencionar los aportes de H.I. Ansoff, M. Porter, H. Minzberg, P Lorange, G. Steiner, entre los principales.

Sin embargo no es sino en años recientes que se ha dado un salto cualitativo en lo referente a la dirección estratégica de las organizaciones, por dos razones: La primera, por el desarrollo de tecnologías para el manejo de grandes bases de datos y la inteligencia estratégica, surgiendo diversos conceptos (datawarehousing, datamining, CRM, ERP, entre otros) y la segunda, con el desarrollo conceptual cada vez más sistemico del modelo organizacional que practican los expertos en estrategia, lo cual permite una visión integral de la misma.

La idea de la visión global de la organización llevó a que en 1992, los Profesores Kaplan y Norton desarrollasen el concepto de Cuadro de Mando Estratégico (Balanced Scorecard) como un medio para la gestión estratégica que integren diversos aspectos de las organizaciones en dicho análisis. Sin embargo, años antes de ello, algunos de los sistemistas mencionados, ya habían dado aportes sobre el particular, como es el caso de Beer, con su Proyecto Cybersin, para la dirección estratégica de la economía chilena, los aportes de Checkland, con la aplicación de su Soft Systems Methodology, en la dirección estratégica de empresas, como el caso de la compañías transnacionales Shell (Holanda) o Imperial Chemical Industrias (ICI) (Inglaterra), o el caso de Forrester, con sus contribuciones en Industrial Dynamics, o el enfoque del Prof. Warfield en Interactive Management, aplicado con éxito en el resurgimiento de la Ford Motor Corp. (USA) entre otros. Aplicaciones que han aparecido en diversas presentaciones hechas en encuentros académicos, libros y artículos publicados en diversas revistas especializadas.

Dichos enfoques son distintos al propuesto por Kaplan y Norton, pues se apoyan en marcos metodológicos sistémicos $y$ cibernéticos, pero que en sí, tienen la misión de obtener una visión sistémica de las mismas y están orientados al desarrollo de marcos de trabajo para dirigir y controlar la gestión estratégica de las organizaciones.

\section{LA PROSPECTIVA TECNOLÓGICA}

\section{Definiendo Prospectiva Tecnológica}

Los Ejercicios de Prospectiva Tecnológica son tentativas sistemáticas para observar a largo plazo el futuro de la 
Ciencia, la Tecnología, la Economía y la Sociedad, con el propósito de identificar las tecnologías emergentes que probablemente produzcan los mayores beneficios económicos y sociales.

La prospectiva tecnológica consiste en hacer pronósticos de escenarios futuros de la demanda de bienes o servicios, pero sin tomar en cuenta necesariamente las tendencias como en el caso de otros métodos de predicción que se realizan contando con el apoyo de herramientas estadísticas tipo la econometría.

Los escenarios futuros se construyen en función de la opinión que dan grupos de expertos de cada sector. Los expertos son seleccionados rigurosamente en base a su experiencia profesional en cada tema bajo estudio, y provienen tanto del sector público, como del sector privado y la Academia. A cada uno de ellos se les proporciona una batería de preguntas, cuya estructura y secuencia permitirán que a través de las respuestas se puedan vislumbrar los posibles escenarios futuros que tendrán que enfrentar las diferentes cadenas productivas.

Por la naturaleza e importancia de sus resultados, la Prospectiva Tecnológica es ampliamente empleada en la formulación de Políticas Públicas y Empresariales en los países desarrollados y en las NICs.

\section{Cómo se ejecuta la Prospectiva Tecnológica}

Dada su complejidad, la Prospectiva Tecnológica exige crear un mecanismo al interior de los países que permita la ejecución sistemática y coherente de sus actividades durante un plazo que varía entre $3 y$ 5 años. Este mecanismo se denomina Ejercicio Nacional de Prospectiva Tecnológica.

Cada Ejercicio Nacional realiza el Estudio de entre 3 y 8 Áreas Temáticas, en función de las prioridades de cada economía. Un Ejercicio Nacional completo puede realizarse una vez por cada década.

\section{La Prospectiva Tecnológica en el mundo}

En los países industrializados esta metodología de planificación se viene implementando desde los años cincuenta. sienac los pioneros Japón y Estados Unidos. Gran parte del desarrollo tecnológico de estos pałses y de Europa se ha debido a su capacidad de identificar las demandas futuras de los mercados, aún cuando ni los propios consumidores eran conscientes de los productos o servicios que demandarían en los próximos años.

En los últimos años, el empleo de la Prospectiva Tecnológica ha permitido a medianas economías tener altas tasas de crecimiento económico, como en el caso de Irlanda, cuyo PBI se ha duplicado en menos de una década, luego de la ejecución de su Primer Ejercicio Nacional de Prospectiva Tecnológica.

En el caso de Latinoamérica, esta metodología se comenzó a utilizar desde hace dos años por iniciativa de la Organización de las Naciones Unidas para el Desarrollo Industrial (ONUDI), quien puso en marcha su Programa Regional de Prospectiva Tecnológica para América Latina y el Caribe. De esta manera, México, Argentina, Brasil, Chile, Colombia, Bolivia y Uruguay han iniciado sus Programas Nacionales de Prospectiva Tecnológica, con significativo éxito.

\section{Etapas de un Ejercicio de Prospectiva Tecnológica}

Un Ejercicio de Prospectiva Tecnológica orientado al futuro de las PYMEs debería tener los siguientes pasos:

\section{SELECCIÓN DEL EQUIPO RESPONSABLE DEL ESTUDIO:}

Alrededor de diez profesionales con amplia experiencia en la asesoría empresarial a PYMEs, tanto en aspectos de gestión, tecnología, comercialización y financiamiento. 


\section{Planeamiento del edercicio:}

Esta etapa comprende una serie de actividades correlacionadas entre sí:

- Cronograma del Ejercicio: Tiene como principal restricción el tiempo en el cual se requieren que los resultados se encuentren disponibles. Un Ejercicio de Prospectiva Tecnológica dura aproximadamente 8 meses, desde su inicio hasta la publicación de sus resultados.

- Definición del kit de herramientas metodológicas a emplear: Existen más de 100 metodologías que se pueden emplear en un Ejercicio de Prospectiva Tecnológica. De ellas, las más empleadas son:

* Exploración del Entorno

* Análisis de Tendencias

* Análisis Morfológico

* Arboles de Pertinencia

* Encuesta Delphi

* Análisis de Jerarquía de Procesos

* Matriz de Impacto Cruzado

* Planeamiento de Escenarios

- Participantes del Ejercicio: Se debe establecer el número y perfil de los participantes de los ejercicios que se llevarán a cabo en cada metodología. Esto nos permitirá identificarlos, buscarlos y comprometerlos a que participen.

- Identificación de las fuentes de información: Un Ejercicio de Prospectiva Tecnológica sobre el futuro de las PYMEs debe tener acceso a un número muy significativo de fuentes de información nacionales e internacionales sobre todos los aspectos vinculados con la operación de las PYMES.

- Presupuesto del Ejercicio: El cronograma, las metodologías, las fuentes de información y la participación de los agentes involucrados (stakeholders) dependen en gran medida del Presupuesto que se disponga para el Ejercicio. Dada la magnitud e importancia de un Ejercicio de Prospectiva Tecno- lógica sobre el futuro de las PYMEs, se estima que el presupuesto estaría alrededor de US $\$ 80,000$.

\section{EJECUCIÓN DEL EJERCICIO:}

El Grupo de Tarea responsable de la ejecución del Ejercicio tendrá que identificar a partir de sus fuentes primarias y secundarias de información los principales factores de cambio (drivers) que influyen directa $e$ indirectamente sobre el futuro de las PYMEs. En ese sentido dada la amplitud de las actividades de las PYMEs en el Perú, se sugiere emplear, en la medida de lo posible, un enfoque de Cadenas Productivas: Cadena Productiva Textil-Confecciones, Cadena Productiva Minero-MetalúrgicaMetalmecánica, Cadena Productiva Madera-Muebles o Cadena Productiva Agro-Agroindustrial.

Entre otras, a las que habría que agregar actividades transversales, tales como: Servicios o Artesanía.

\section{Difusión DE LOS RESULTADOS:}

Una vez validados los resultados de la aplicación de las distintas metodologías, que no son sino una serie de escenarios futuros probables, se debería realizar un extensivo programa de difusión de los mismos, a fin que los tomadores de decisiones (Gobierno, Empresarios y la Academia) puedan planear las mejores estrategias para aprovechar las oportunidades que los escenarios futuros identificados pueden tener para las PYMEs peruanas.

\section{¿QUÉ SE ESPERA CONSEGUIR?}

Un Ejercicio de Prospectiva. Tecnológica sobre el futuro de las PYMEs al año 2010 nos permitiría:

1. Identificar, por un lado, las actividades con mayor potencial de éxito futuro; $y$ por otro lado, las actividades que ya se encontrarían en declinación al finalizar la década. Esta información facilitaría 
la toma de decisiones sobre el destino de las inversiones futuras. Cada año, los empresarios invierten más de US\$ 400 millones en actividades que no logran culminar con éxito en el mercado. Es una pérdida enorme de recursos $y$ ahorros, en los que las familias ponen en riesgo su estabilidad. Reducir el riesgo es un motivo más que suficiente para hacer Prospectiva Tecnológica en el país.

2. Conocer las principales fuerzas que generan el cambio en los distintos mercados de las PYMEs, con la finalidad de desarrollar las ventajas competitivas que hagan sostenible su crecimiento. El nuevo paradigma de esta década ya no es ni la eficiencia ni la competitividad, es la sostenibilidad, y para que una empresa sea sostenible en el tiempo, necesita más que nunca desarrollar ventajas competitivas dinámicas basadas en la investigación tecnológica y en la innovación.

3. Orientar los esfuerzos emprendedores de los jóvenes hacia las nuevas actividades y mercados emergentes. Los nuevos espacios que se vienen creando requieren nuevas visiones y enfoques que los jóvenes pueden aportar. La principal restricción que enfrentan es la falta de información relevante sobre el futuro que les tocará vivir. Un Ejercicio de Prospectiva Tecnológica les entregaría esa información que necesitan.

Si un Ejercicio de Prospectiva Tecnológica orientado al futuro de las PYMEs en el 2010 resultaría tan útil, ¿por qué no nos comprometemos a realizarlo?

\section{CUADRO DE MANDO ESTRATÉGICO (BALANCED SCORECARD)}

\section{¿Qué es Cuadro de Mando Estratégico (Balanced Scorecard)?}

El Cuadro de Mando Estratégico es un enfoque que plantea ver a la organización desde 4 perspectivas distintas e interrelacionadas:
- La Perspectiva desde el Punto de Vista Financiero

- La Perspectiva desde el Punto de Vista del Cliente

- La Perspectiva de los Procesos Internos de la Organización

- La Perspectiva de la Innovación, Desarrollo y el Crecimiento.

La Fig. N..$^{\circ} 1$, muestra estas cuatro perspectivas:

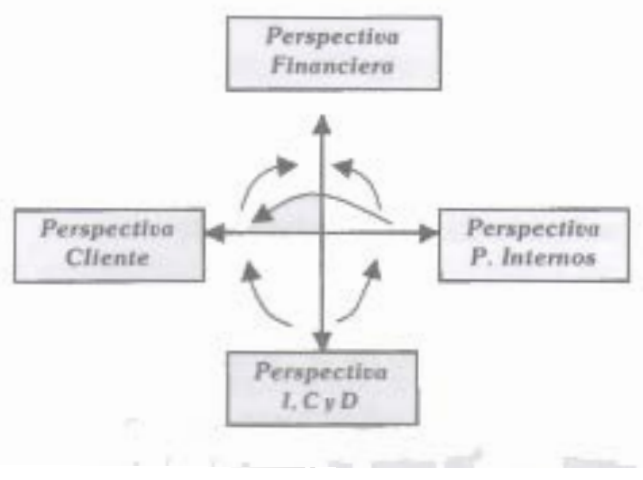

Fig. No. 1: Las 4 Perspectivas del Balanced Scorecard

La idea es que dentro de estas visiones se establezcan diversos indicadores de gestión. que permitan hacer un seguimiento adecuado del desempeño en cada una de estas perspectivas y sus relaciones con las demás y en consecuencia, lograr un sistema de medición integral y permanente del desempeño de la organización como un todo; integrando a la vez, la misión, visión, objetivos, iniciativas estratégicas, sus indicadores de gestión y las metas a cumplir.

En otras palabras, la idea es ver cómo se puede concebir e implantar un proceso estratégico que se inicia en el cumplimiento de la misión, visión, objetivos y metas, pasando por el cumplimiento de un conjunto de iniciativas estratégicas, para culminar en el seguimiento en el tiempo de los distintos indicadores pertenecientes a las cuatro perspectivas indicadas, con la finalidad de implantar un proceso permanente de gestión estratégica. 


\section{La Perspectiva desde el Punto de Vista FINANCIERO}

La perspectiva desde el punto de vista financiero tiene por objetivo hacer un seguimiento de la gestión financiera de la organización a fin de manejar cuatro aspectos fundamentales: Liquidez, Solvencia, Rendimiento y Actividad

Entre los indicadores que nos muestran la evolución de la liquidez de la organización pueden mencionarse:

- Prueba Ácida

- Liquidez

Entre los indicadores que muestran la evolución de la solvencia están:

- Relación Deuda/Capital

- Cubrimiento de la Deuda

Entre los indicadores que permiten tener una idea dei rendimiento están.

- El ROS (Retum on Sąles)

- El ROI (Return on lnvestment)

- El ROE (Return on Equity)

- El ROCE (Return on Capital Employed)

- EVA (Economic Value Added)

Entre los indicadores que se estudian para analizar los ratios de actividad de la organización, se pueden mencionar a:

- Rotación de Activos Fijos

- Rotación de Inventarios

- Rotación de Cuentas por Cobrar

- Período Promedio de Cobro

Básicamente representa la visión pasado-presente de la organización.

\section{La Perspectiva desde el Punto de Vista del Cliente}

La perspectiva del cliente tiene por objetivo ponerse en la posición de a quiénes sirve la organización y analizar su impacto en la satisfacción de sus necesidades. Desde esta perspectiva es posible establecer un conjunto de indicadores que pueden permitir evaluar la gestión de la organización en el cumplimiento de satisfacer las necesidades de los clientes.

Entre los principales indicadores podrán mencionarse:

- Cobertura de mercado

- Número de reclamos

- Número de devoluciones

- Reincidencia del cliente por nuestros servicios

- Imagen del producto o servicio

- Imagen de la organización

- Volumen de ventas

- Número de clientes

- Tipos de clientes

- Grado de satisfacción de los clientes

- Fidelidad del cliente

- Retención del cliente

Representa una visión del presente de la organización.

\section{La Perspectiva de los Procesos Inter- NOS de la ORganización}

La visión de los procesos de la organización está relacionada con el estudio de indicadores que midan el impacto en la mejora, cambios, inclusión/eliminación de los procesos de gestión de la organización. La idea en esta visión es que se tengan indicadores que permitan evaluar cuáles son los procesos críticos que dan valor agregado al producto o servicio que se brinda y ver su evolución en el tiempo. Esto tiene que ver con la cadena de valor de la organización.

Los procesos que se evalúen pueden ser de carácter estratégico, táctico e inclusive operativo. En concreto los indicadores que se plantean para esta visión tienen que ver 
con la medición de la calidad de los procesos y el ciclo temporal de cada uno de ellos.

Lo que se busca es básicamente ser eficaces y eficientes en la ejecución de los procesos internos. Lo primero tiene que ver con un alineamiento de la misión, visión y objetivos con los procesos y la cadena de valor de la organización; lo segundo tiene que ver con el adecuado y óptimo empleo de los recursos. De manera que los indicadores de esta perspectiva vienen de allí. Básicamente representa el quehacer actual de la organización, conjuntamente con la perspectiva de clientes

\section{La Perspectiva de la Innovación, Desa- rrollo y el. Crecimiento}

La perspectiva de la innovación, el desarrollo y el crecimiento permite a la organización, establecer un conjunto de objeti vos e indicadores en relación a lo por hacer en ella. Es decir que se trata de indicadores que miran hacia el presente y futuro de la organización.

Aquí pueden estar indicadores que tienen que ver con medir el impacto de los procesos de capacitación de personal, los nuevos proyectos de innovación tecnológica de la organización, el mejoramiento en la imagen de la organización, el impacto de una visión compartida, el valor del conocimiento del mercado, la importancia del dominio de un nicho de conocimiento determinado o la importancia de tener una red de conexiones a nivel internacional. En suma están los indicadores que tienen que ver con lo aquello que tendrá un impacto futuro en la organización, usualmente relacionado con la tecnología, innovación y el factor humano.

\section{El Cuadro de Mando (Balanced Scorecard): Herramienta para el Crecimiento y Desarkollo de las PYMEs}

El Cuadro de Mando Estratégico (Balanced Scorecard), luego de un período de pruebas en diversas organizaciones norteamericanas y de otros contextos en un período comprendido entre 1992 y 1996, fue lanzado a la palestra, como una herramienta que serviría para la gestión estratégica de las organizaciones. De esa fecha al presente, podría decirse que efectivamente, el Cuadro de Mando sintetiza muchas cosas que un gerente o un planeador estratégico desearía ver de una organización, para determinar su crecimiento y desarrollo.

De allí que se esté convirtiendo en un marco de trabajo que sintetizando lo pasado con lo presente y futuro de la organización, mediante la combinación de las 4 perspectivas planteadas, permita tener una concepción amplia y cuasi completa de la misma, en relación con su entorno.

Sobre su aplicación, puede mencionarse de su empleo cada más creciente en el contexto mundial y concretamente en el latinoamericano, donde se le viene aplicando con el soporte y empleo de distinta tecnología.

A través de los miembros de la Sociedad Latinoamericana de Estrategia (SLADE), con sede en Sao Paulo, se han realizado congresos y seminarios en distintas capitales de Latinoamérica en donde en concepto de Balanced Scorecard (BSC) ha sido uno de los temas con frecuencia tocados. Igualmente, puede mencionarse el enfoque sistémico que se la ha dado al BSC, a través de los trabajos del Instituto Andino de Sistemas - IAS, en donde haciendo empleo de una tecnología y metodología adhoc, denominada Metodología Sistémica para la Elaborar y Mantener un Cuadro de Mando (Balanced Scorecard), con los apoyos de la tecnología de simulación en Dinámica de Sistemas, ha permitido crear aplicaciones concretas en distintos sectores de actividad, tanto en ámbito nacional como en el extranjero, planteando un enfoque que se conoce como Dynamics Balanced Scorecard (Balanced Scorecard Dinámico), considerando aplicaciones susceptibles de 
poder analizar diversos escenarios de desenvolvimiento, a través de simulaciones de desenvolvimiento futuro de la empresa, en el computador y sobre esa base plantear el accionar futuro de la organización.

En este sentido, consideramos que el Batanced Scorecard, provisto de la tecnología pertinente, y que existe al alcance de las PYMEs latinoamericanas, puede convertirse en un instrumento que podría ser la palanca para el crecimiento y desarrollo de las PYMEs, al permitir contar con una tecnología que visualizar posibles oportunidades y amenazas futuras, considerando a la vez, la evolución de sus fortalezas y debilidades en el tiempo, al contar en un entorno tecnológico que permite visualizar las diversas alternativas de su accionar futuro, a inversiones muy asequibles a la realidad de las PYMEs.

El enfoque de Dynamics Balanced Scorecard mencionado previamente, podría ser la forma de analizar opciones "what if" ("que pasaría si..") para las PYMEs, considerando distintos escenarios tanto de la situación interna como externa de las mismas, permitiendo visualizar las diversas consecuencias de los potenciales acontecimientos, lo cual debe llevar al aprendizaje en la gestión estratégica y la mejora en la calidad de decisiones, teniendo como laboratorio de trabajo a la computadora, en vez de experimentar dichas decisiones en el mundo real.

Todo ello debe llevar a significativos ahorros financieros, de energía física, mental, emocional, mejorando el desempeño general de las PYMEs y su impacto en su ámbito socio - económico.

\section{CONCLUSIONES}

Se puede concluir entre otras cosas que el Cuadro de Mando (Balanced Scorecard) tiene las siguientes ventajas para las PYMEs:
- Puede constituirse en un instrumento de gran relevancia para el control integral de las PYMEs en su relación con el entorno, que conecta la trayectoria pasada, con el presente y futuro de la organización.

- Es un útil intelectual que replanteando el enfoque tradicional de analizar la organización desde indicadores contable-financieros, considera otros indicadores que permiten conectar la misión, visión y objetivos de la misma con las estrategias $e$ indicadores que delinean la ruta a seguir en el futuro de la organización, que aplicado a las PYMEs, traería como consecuencia una nueva y más amplia y actualizada forma de comprender la problemática de las mismas y mejorar por ende su manejo estratégico.

- Conforma un medio para obtener una visión compartida entre los trabajadores de la organización acerca de los problemas que tiene la misma y lo por hacer en el futuro a fin de plantear un crecimiento y desarrollo saludable $y$ continuo, lo cual permite una mayor integración en el trabajo del grupo directriz de las PYMEs, apuntando todo ello a tener una visión compartida tanto en la comprensión de los problemas, como en su solución.

- Permite considerar adecuadamente distintos aspectos sumamente importantes tanto del entorno como del interno de la organización (los clientes externos e internos) así como valorar aquello que en estos tiempos de la era de la información es mucho más importante que los activos tangibles: los activos intangibles que vienen hacer las habilidades del personal con que cuenta la organización, la información, el know-how, las relaciones humanas, organizacionales y con el mercado. Esto puede ser estudiado formalmente con el BSC, analizando su impacto en 
el crecimiento y desarrollo de los indicadores de la perspectiva financiera.

- Sin embargo, es menester mencionar también, que es posible, desde una perspectiva sistémica amplia, y pensando en aplicaciones más allá de las organizaciones empresariales, replantear la propuesta de Kaplan y Norton, considerando otras visiones, además de las cuatro propuestas, que permitan comprender más ampliamente la problemática del interno y el entorno del sistema social considerado, tal como nuestra experiencia profesional nos indica, basada en proyectos de índole social, de seguridad ciudadana y de desarrollo sostenible de regiones $y$ países elaborados en estos años.

- Finalmente puede concluirse que existe tecnología asequible a los niveles de inversión de las PYMES, que abre las posibilidades de aplicar las bondades de la aplicación del Balanced Scorecard en las PYMES, inversión que rápidamente podría ser recuperada, con los beneficios que podrían obtener las mismas, a partir de una mejora substancial en su dirección estratégica.

\section{ANEXO \\ Metodologías empleadas en los Ejer- cicios de Prospectiva Tecnológica \\ Los Ejercicios de Prospectiva Tecnoló- gica emplean una serie de metodologías para identificar escenarios futuros. Las más empleadas son:}

\section{Método Delphi}

Consiste en preguntarle al grupo de expertos sus opiniones (juicios de valor basados en conocimiento, experiencia, imaginación, sentido común e intuición), acerca del comportamiento a futuro de un grupo dado de variables a indicadores; con la finalidad de tener una idea lo más clara posible de la situación futura descrita a través de los mismos.

\section{Método de Probabilidades de Bayes}

Aplicación de las fórmulas derivadas del Teorema de Bayes a la determinación de las llamadas probabilidades revisadas; asociadas a un conjunto dado de hipótesis (escenarios factibles de presentarse) mutuamente excluyentes, como consecuencia de las evidencias (hechos) observados.

\section{Método de la Matriz de Impacto cruzado}

Su lógica básica subyacente, consiste en hacer una exploración del futuro (prospectiva) sobre la base de una serie de eventos que pueden o no ocurrir dentro de un horizonte temporal considerado. En tal sentido, el vocablo o termino "evento» se refiere aquí a una hipótesis que puede o no ser cierta, según que tal evento ocurra o no en el marco temporal analizado.

\section{Método de Análisis de Jerarquía de Procesos}

Aunque la "AHP" es una técnica "focalizada fundamentalmente" como herramienta de apoyo a la toma de decisiones se la ha impulsado como una técnica que da apoyo también a otros problemas de naturaleza intrínsecamente no-estructurada, como lo son la modelación y el análisis de conflicto y el análisis prospectivo; en particular, como técnica de pronóstico.

\section{Método de Análisis Morfológico}

Como objeto, la técnica persigue explorar todas las posibilidades a que pueda evolucionar un sistema determinado. Para ello, es necesario identificar con gran precisión lo que se denominan los parámetros caracterizadores del sistema (o tecnología) 
bajo estudio. Dentro de este orden de ideas, al análisis morfológico le concierne el desarrollo de aplicaciones prácticas, que nos permitirán descubrir y analizar lo estructural o interrelaciones morfológicas entre fenómenos, objetos o conceptos; para así usar los resultados obtenidos, en la construcción de nuevos sistemas o en la visualización de nuevas formas en los sistemas sociales, económicos y políticos de nuestras sociedades.

\section{BIBLIOGRAFÍA}

- Profesor S. Beer. Modelo de Sistema Viable.

- Profesor P.B. Checkland. La Metodología de Sistemas Blandos (Soft Systems)

- Profesor J. H. Warfield. Aportes en Interactive Management
- J. Forrester y P. Senge. La Dinámica de Sistemas y las 5 Disciplinas

- R. Ackoff. Strategic Interactive Management

- H.I. Ansoff, M. Porter, H. Minzberg, P. Lorange, G. Steiner. En el campo del pensamiento estratégico.

- Fernando Ortega San Martín. CONCYTEC Jefe de la Oficina de Innovación y Prospectiva Tecnológica.

- Ricardo A. Rodríguez Ulloa. Presidente, Instituto Andino de Sistemas-IAS, Soft System Dynamics Methodology - SSDM, producto de la fusión de la Soft Systems Methodology- SSM y de la Dinámica de Sistemas-SD y de la Metodología Sistámica para Elaborar y Mantener un Cuadro de Mando (Balanced Scorecard) (MSBSC-EM). 